\title{
Sustained local application of epidermal growth factor to accelerate reepithelialization of tracheal grafts
}

Jinbo Zhao, MD,* Yong Han, MD,* Zhibo Liang, MD, Zhipei Zhang, PhD, Qiang Lu, MD, Xiaolong Yan, MD, and Xiaofei Li, MD

Objective: Epidermal growth factor-loaded gelatin microspheres were tested for potential to accelerate tracheal allograft reepithelialization.

\begin{abstract}
Methods: Epidermal growth factor-loaded gelatin microspheres were prepared by optimal double-phase emulsified condensation polymerization. One hundred age-matched allogeneic mice were randomly allocated to local administration of $1 \mathrm{mg}$ epidermal growth factor-loaded gelatin microspheres (containing $1 \mu \mathrm{g}$ epidermal growth factor), $1 \mathrm{mg}$ gelatin microspheres, $1 \mu \mathrm{g}$ epidermal growth factor, or nothing (control, all groups $\mathrm{n}=25$ ) during orthotopic transplant of C57BL/6 donor tracheal segments into BALB/c recipients without immunosuppressors. On days 7, 14,21,35, and 52 after transplant, 5 mice per group were killed and evaluated by histologic assessment and scanning electronic microscopy for reepithelialization and fibrosis of tracheal grafts.
\end{abstract}

Results: Mean diameter of gelatin microspheres was $107 \mu \mathrm{m}$. Microspheres could not be fully degraded until 35 days after transplant in vivo. On days 7, 14, and 21, epithelium score and ratio of lamina propria to tracheal cartilage were not statistically different between mice with epidermal growth factor-loaded gelatin microspheres and other groups. On days 35 and 52, however, epithelium score was higher and ratio of lamina propria to tracheal cartilage was lower in epidermal growth factor-loaded gelatin microsphere recipients; these mice also had almost complete differentiation of regenerated epithelium into ciliated columnar epithelium on days 35 and 52, earlier than in other groups.

Conclusions: Gelatin microspheres act as a functional vector for epidermal growth factor. Sustained local application of epidermal growth factor could accelerate reepithelialization of tracheal allografts. (J Thorac Cardiovasc Surg 2010;140:209-15)

Tracheal transplant has for many years been studied as a means of repairing extensive defects of the trachea. Although experimental transplants have been performed on animals and even human patients, ${ }^{1}$ problems such as graft rejection, ischemia, and airway obstruction have resulted in graft failure.

Reepithelialization plays an essential role in viability of tracheal grafts. Because the epithelium of donor has been thought to be the primary target of allograft rejection, ${ }^{2}$ reepithelialization of orthotopic tracheal grafts with recipientderived epithelium may be a great help in preventing airway obliteration and rejection. ${ }^{3-5}$ Tracheal reepithelialization takes a long time, ${ }^{6}$ however, so finding a way to accelerate

\footnotetext{
From the Department of Thoracic Surgery, Tangdu Hospital, Fourth Military Medical University, Xi'an, Shaanxi, China.

Supported by National Nature Science Foundation of China No. 39970707.

Disclosures: None.

*These authors contributed equally to this work.

Received for publication Aug 15, 2009; revisions received Oct 13, 2009; accepted for publication Oct 25, 2009; available ahead of print Dec 28, 2009.

Address for reprints: Xiaofei Li, MD, Department of Thoracic Surgery, Tangdu Hospital, Fourth Medical Military University, Xinsi Rd, Xi'an, Shaanxi 710038, China (E-mail: lxfchest@fmmu.edu.cn).

$0022-5223 / \$ 36.00$

Copyright (c) 2010 by The American Association for Thoracic Surgery

doi:10.1016/j.jtcvs.2009.10.036
}

the epithelium regeneration may contribute to the success of tracheal transplant.

Epidermal growth factor (EGF) could promote epithelial tissue growth, proliferation, and differentiation. ${ }^{7}$ It has been used clinically as an activator to accelerate wound healing, with satisfactory outcome. ${ }^{8}$ If EGF could be administered to recipients who have received tracheal transplants, it might be useful in accelerating tracheal reepithelialization. The half-life of EGF is so short that it has to be applied several times a day to make ensure functionality, however, which impedes clinical use. A vector that could load and continuously release active EGF is therefore needed.

In this study, gelatin microspheres (GMs) were used as vectors to load EGF (EGF-GMs). EGF was thus applied locally and persistently to tracheal grafts to observe its effect on the reepithelialization and fibrosis of the tracheal grafts.

\section{MATERIALS AND METHODS \\ Preparation of GMs and Characterization \\ Examination}

The GMs were prepared by optimal double-phase emulsified condensation polymerization. The gelatin (Sigma, St Louis, Mo) solution $(20 \%, 6$ $\mathrm{mL}, 0.3 \mathrm{ml}$ polysorbate 80 included, analytic reagent; Xi'an Chemical Co. Xi'an, China) was slowly dropped into preheated liquid paraffin (50 $\mathrm{mL}, 50^{\circ} \mathrm{C}$, analytic reagent, Xi'an Chemical) with constant stirring (500 


$$
\begin{aligned}
& \text { Abbreviations and Acronyms } \\
& \begin{aligned}
\text { EGF } & \text { epidermal growth factor } \\
\text { EGF-GM }= & \text { epidermal growth factor-loaded gelatin } \\
& \text { microsphere } \\
\text { ES } & \text { epithelium score } \\
\text { GM }= & \text { gelatin microsphere } \\
\text { LCR } & \text { ratio of lamina propria to tracheal } \\
& \text { cartilage } \\
\text { PBS } \quad= & \text { phosphate-buffered saline solution }
\end{aligned}
\end{aligned}
$$

$\mathrm{rpm}$ ) for 10 minutes and then quickly cooled down to $0^{\circ} \mathrm{C}$. Five minutes after stirring, the formed microspheres were dewatered by adding isopropanol $(10 \mathrm{~mL}$, analytic reagent; Xi'an Chemical). The GMs were washed with isopropanol and ethyl ether (analytic reagent; Xi'an Chemical) 3 times to eliminate the remaining paraffin. After the ethyl ether had volatilized, the particles were added into $2.5 \%$ glutaraldehyde $\left(4^{\circ} \mathrm{C}\right.$, analytic reagent; Xi'an Chemical) aqueous solution for crosslinking. Twenty-four hours later, $2 \%$ glycine solution (Sigma) was used to stop the reaction. The GMs were freeze-dried and preserved for further use.

\section{Preparation of EGF-GMs}

The GMs were exposed to cobalt 60 for sterilization. A 25-mg portion of GMs was immersed into $125 \mu \mathrm{L}$ phosphate-buffered saline solution (PBS, $\mathrm{pH}$ 7.4) containing $25 \mu \mathrm{g}$ EGF (PeproTech, Inc, Rocky Hill, NJ) and shaken for 2 hours under sterile conditions. The GMs loaded with EGF were collected, freeze-dried, and preserved for further use.

\section{Characterization Examination of GMs In Vitro and In Vivo}

The particle size was analyzed with a laser diffraction particles size analyzer (Masterizer 2000; Malvern Instruments Ltd, Malvern, UK). The characteristics of microspheres in vitro were examined by scanning electron microscopy (S-3400 N; Hitachi, Ltd, Tokyo, Japan). The GMs were weighed before and after being put into PBS ( $\mathrm{pH} 7.4$ ) for 2 hours. The swelling ratio was derived as the weight of GMs put in PBS for 2 hours divided by the dry weight of the GMs.

During tracheal transplant, the EGF-GMs or GMs were locally applied. On days 7, 14, 21, 35, and 52 after tracheal transplant, the residue of the EGF-GMs or GMs was retrieved when the animals were sacrificed. The degradation of the microspheres in vivo was examined with light microscopy.

\section{Animal Groups}

One hundred age-matched allogeneic mice (C57BL/6 as donor and $\mathrm{BALB} / \mathrm{c}$ as recipient; Experimental Animal Center, The Fourth Military Medical University, Xi' an, China) were randomly divided into EGF-GM recipient, GM recipient, EGF recipient, and control groups $(n=25$ each). In the EGF-GM and GM groups, the outer surfaces of tracheal grafts after transplant, including the anastomoses, were covered circumferentially with $1 \mathrm{mg}$ EGF-GMs containing $1 \mu \mathrm{g}$ EGF or $1 \mathrm{mg}$ GMs without EGF. In the EGF group, the outer surfaces of tracheal grafts after transplant, including the anastomoses, were soaked circumferentially with $1 \mu \mathrm{g}$ EGF diluted with $20 \mu \mathrm{L}$ PBS). In the control group, nothing was administered to any graft.

All animals received humane care in compliance with the guidelines of the Fourth Military Medical University for animal experiments, which met the standards of the Principles of Laboratory Animal Care formulated by the National Society for Medical Research and the Guide for the Care and the Use of Laboratory Animals (www.nap.edu/catalog/5140.html) prepared by the Institute of Laboratory Animal Resources and published by the National Institutes of Health.

\section{Tracheal Grafting Procedures}

The tracheal grafting procedure was performed as described by Genden and colleagues. ${ }^{9}$ Ketamine $(50 \mathrm{mg} / \mathrm{kg})$ and xylazine $(10 \mathrm{mg} / \mathrm{kg})$ were injected subcutaneously to anesthetize the mice. The donor mouse tracheal segment was exposed through an anterior midline neck incision. A 6-ring circumferential tracheal segment (from the 2nd ring to the 7th ring) was separated carefully, excised, and put into a sterilized Petri dish with cooled PBS. The recipient mouse was anesthetized, and the tracheal segment was exposed through an anterior midline neck incision. A 2-ring incision was made in the recipient trachea. The donor graft was orthotopically transplanted into the recipient tracheal defect and secured with 10-0 nylon interrupted transtracheal sutures. The strap muscles and the skin were sewn up with 5-0 nylon sutures. After the operation, recipients were placed under a warming lamp for 3 hours.

\section{Histologic Evaluation}

At 7, 14, 21, 35, and 52 days after tracheal transplant, 5 mice from each group were killed by neck dislocation. The tracheal graft segments were harvested, fixed in buffered formalin $(4 \%)$, and embedded in paraffin. The grafts were then cut longitudinally into $5-\mu \mathrm{m}$ sections and stained with hematoxylin and eosin. The tracheal epithelium of the donor segment after transplant was evaluated. The epithelium was assessed in grades as follows: 0 , no epithelium; 1 , single layer; 2 , stratified epithelium; 3 , normal ciliated columnar epithelium. The length of each grade of epithelium was measured with computer-assisted image technology (Image-Pro Plus 6.0, Media Cybernetics, Inc, Bethesda, Md). The percentage of each grade of epithelium was calculated. The epithelium score (ES) was then calculated by taking the percentages represented by each grade, multiplying these by the respective grade numbers, and adding the results.

The ratio of the lamina propria to the tracheal cartilage (LCR) was used to assess the degree of fibrosis. The thicknesses of the cartilage and the lamina propria were measured with computer-assisted image technology (Image-Pro Plus 6.0; Media Cybernetics). The LCR was calculated and averaged from 5 points for each section.

The histologic evaluation was performed by 2 investigators (L. Gong and S.J. Zhu) blinded to other data. For each graft, 3 random slides were evaluated for the ES and the LCR.

\section{Scanning Electron Microscopy}

The tracheal specimens were harvested and fixed with $3 \%$ glutaraldehyde in PBS for 24 hours, dried, and gold sprinkled in a vacuum. The specimens were then viewed with a scanning electron microscope (S-3400 N; Hitachi). The results were objectively assessed by a pathologist for morphology (X.F. Huang).

\section{Statistical Methods}

Results are expressed as mean $\pm \mathrm{SD}$. Statistical analysis was performed with the nonparametric Kruskal-Wallis 1-way analysis by ranks. SPSS statistical software (version 16.0; SPSS, Inc, Chicago, Ill) was used for all data analysis.

\section{RESULTS}

\section{Characteristics of EGF-GMs In Vitro and In Vivo}

The GMs looked round and regular and were well dispersed both in vitro (Figure 1, A) and in vivo (Figure 1, $B-F)$. The mean diameter was $107 \mu \mathrm{m}$, and the swelling ratio was $6.53 \pm 1.53$. After preservation at $20^{\circ} \mathrm{C}$ to $38^{\circ} \mathrm{C}$ for 2 months, the characteristics of EGF-GMs were unchanged. 

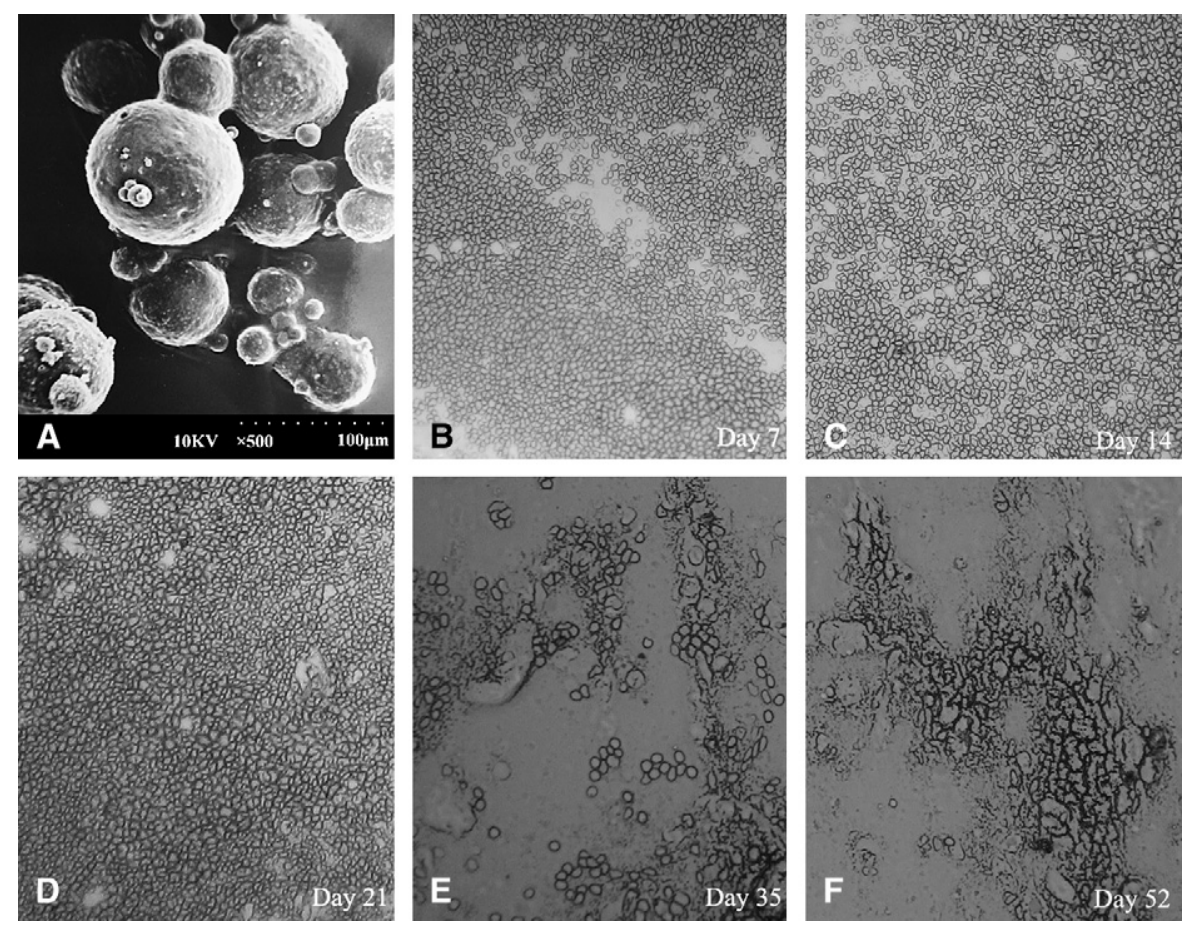

FIGURE 1. Characteristics of epidermal growth factor-loaded gelatin microspheres in vitro were examined with scanning electron microscopy (A), and their degradation in vivo was examined with light microscopy (B-F, original magnification $\times 50$ ). A, Microspheres looked round and regular and were well dispersed in vitro. B through D, microspheres had no distinguishable change in structure or appearance in vivo for 21 days after application. B, Day 7; C, day 14; D, day 21. E, Thirty-five days after application in vivo, most microspheres had lost structure and been degraded. F, Fifty-two days after transplant, microspheres were totally disintegrated.

EGF-GMs had no distinguishable change in structure or appearance when they were locally applied in vivo for 7,14 , or 21 days (Figure 1, $B-D$ ). Thirty-five days after local application in vivo, most of the EGF-GMs had lost structure and been degraded (Figure 1,E). Fifty-two days after transplant, EGF-GMs had been totally disintegrated (Figure 1, F).

\section{EGF-GM Acceleration of Tracheal Graft Epithelial Regeneration}

Histologic examination and ES calculation were used to evaluate the state of tracheal epithelialization. As shown in Figure 2, the ESs of all groups were lowest on day 14 $(P<.05)$, after which they increased gradually. At day 35, the ES in the EGF-GM group had increased quickly, reaching $2.22 \pm 0.09$, whereas the ESs of the other groups were lower $(P<.05)$. Although the ESs of EGF, GM, and control groups had all increased rapidly at day 52, the ES of EGFGMs had reached $2.87 \pm 0.14$, which was still higher than those of the other groups $(P<.05)$.

\section{EGF-GMs Acceleration of Tracheal Graft Epithelial Differentiation}

SEM was used to evaluate the differentiation of tracheal graft epithelium. We found that epithelium in the EGFGM group had differentiated into cilia at 35 days after transplant. SEM showed a dense population of ciliated epithelium in the EGF-GM group, whereas sparse populations of blunted cilia with interspersed mucous cells were seen in other groups (Figure 3). Fifty-two days after transplant, the grafts in the EGF-GM group showed intact, well-differentiated, and functional epithelia (Figure 4, $A$ ). In the EGF, GM, and control groups, the ciliated epithelia were still relatively sparse (Figure 4, $B-D$ ).

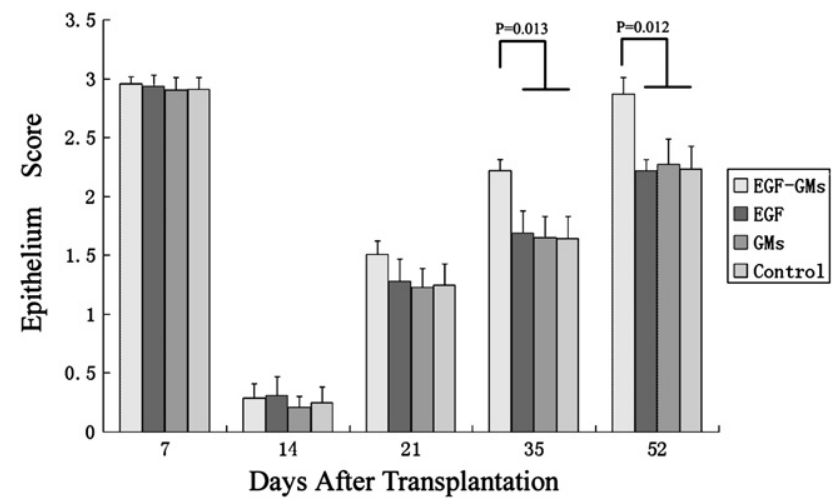

FIGURE 2. State of tracheal epithelium was evaluated by epithelium score. Epithelium score of group treated with epidermal growth factorloaded gelatin microspheres ( $E G F-G M s)$ was higher than scores of groups treated with epidermal growth factor $(E G F)$, gelatin microspheres $(G M s)$, or nothing (Control) on days 35 and 52 after transplant. Error bars represent SD. 

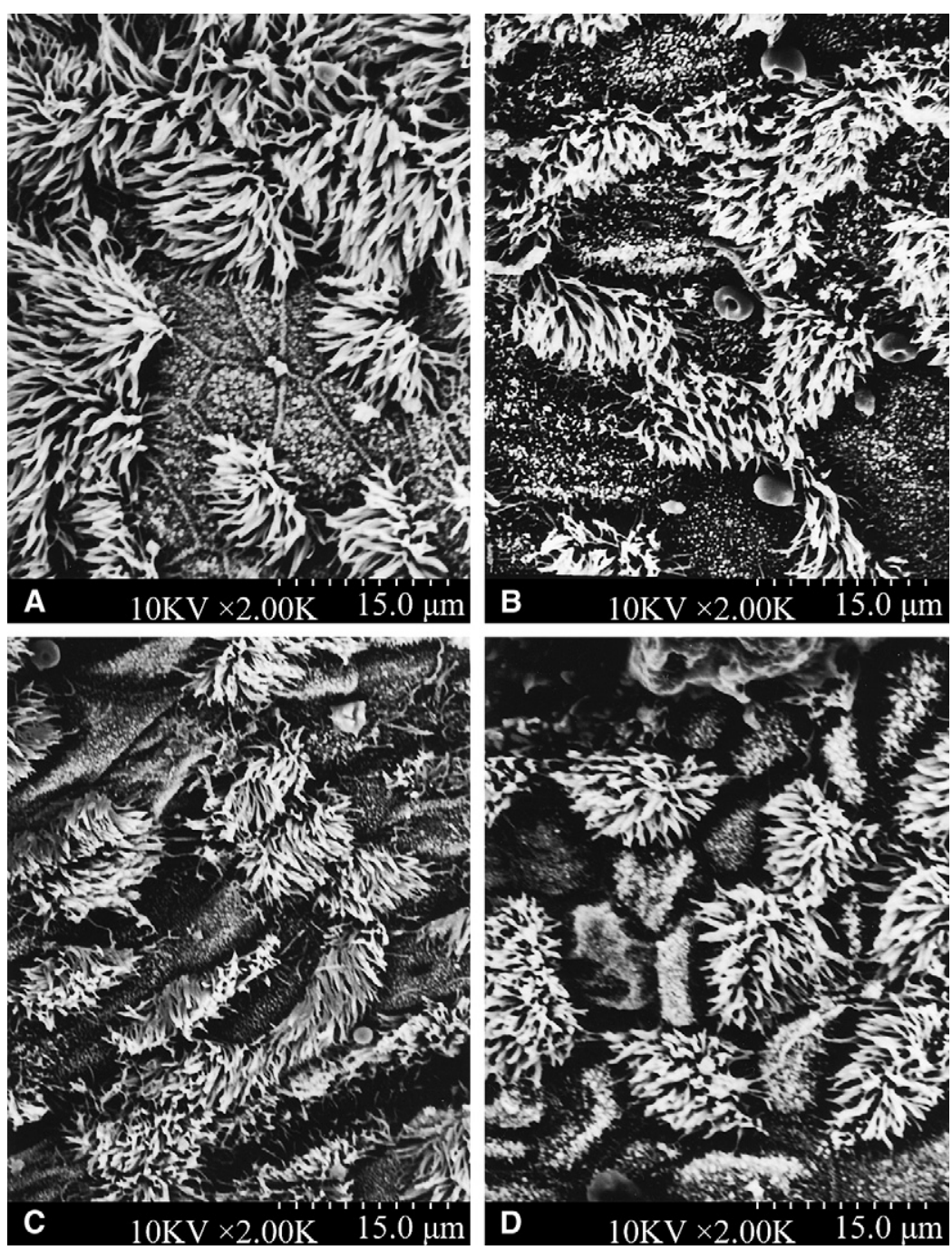

FIGURE 3. Thirty-five days after transplant, scanning electron microscopy showed dense population of ciliated epithelium in epidermal growth factorloaded gelatin microsphere group (A), with sparse populations of blunted cilia with interspersed mucous cells in other groups. B, Epidermal growth factor group; C, gelatin microsphere group; D, control group.

\section{EGF-GMs and Tracheal Grafts Fibrosis}

The LCR was calculated to evaluate the fibrosis of tracheal grafts. On days 7,14 , and 21 , there was no statistical difference among the groups in terms of LCR. On days 35 and 52, the LCRs of the EGF-GM group were $1.44 \pm$ 0.12 and $1.47 \pm 0.12$, respectively, both of which values were lower than those of the other groups $(P<.05$, Figure 5).

\section{DISCUSSION}

Reepithelialization is essential for tracheal grafts. The murine model of orthotopic tracheal transplantation that was adopted in this study has proved to be a good model for experimental study of the reepithelialization of the tracheal graft. ${ }^{3-6}$ With murine orthotopic and heterotopic tracheal transplantation models, it has been proved that immunosuppressors can be withdrawn without inciting acute and chronic rejection if the donor epithelium is progressively replaced by recipient-derived epithelium. ${ }^{4,5}$ It takes at least a 48-day period to complete reepithelialization in immunosuppressed orthotopic tracheal allografts, ${ }^{6}$ however, so it is necessary to find a way to accelerate the epithelial regeneration of the tracheal graft.

Many growth factors have been used in animal models to maintain viability of the tracheal graft. ${ }^{10-18}$ Basic fibroblast growth factor and vascular endothelial growth factor have been studied in tracheal transplants for their strong revascularization effects, and we have also successfully stimulated cartilage regeneration in tracheal grafts with BMP-2. ${ }^{10}$ Although vascular endothelial growth factor was of great benefit to tracheal grafts, it may increase the development of tracheal luminal occlusion through signaling pathways mediated by platelet-derived growth factor to recruit mononuclear inflammatory cells. ${ }^{15,16}$ 

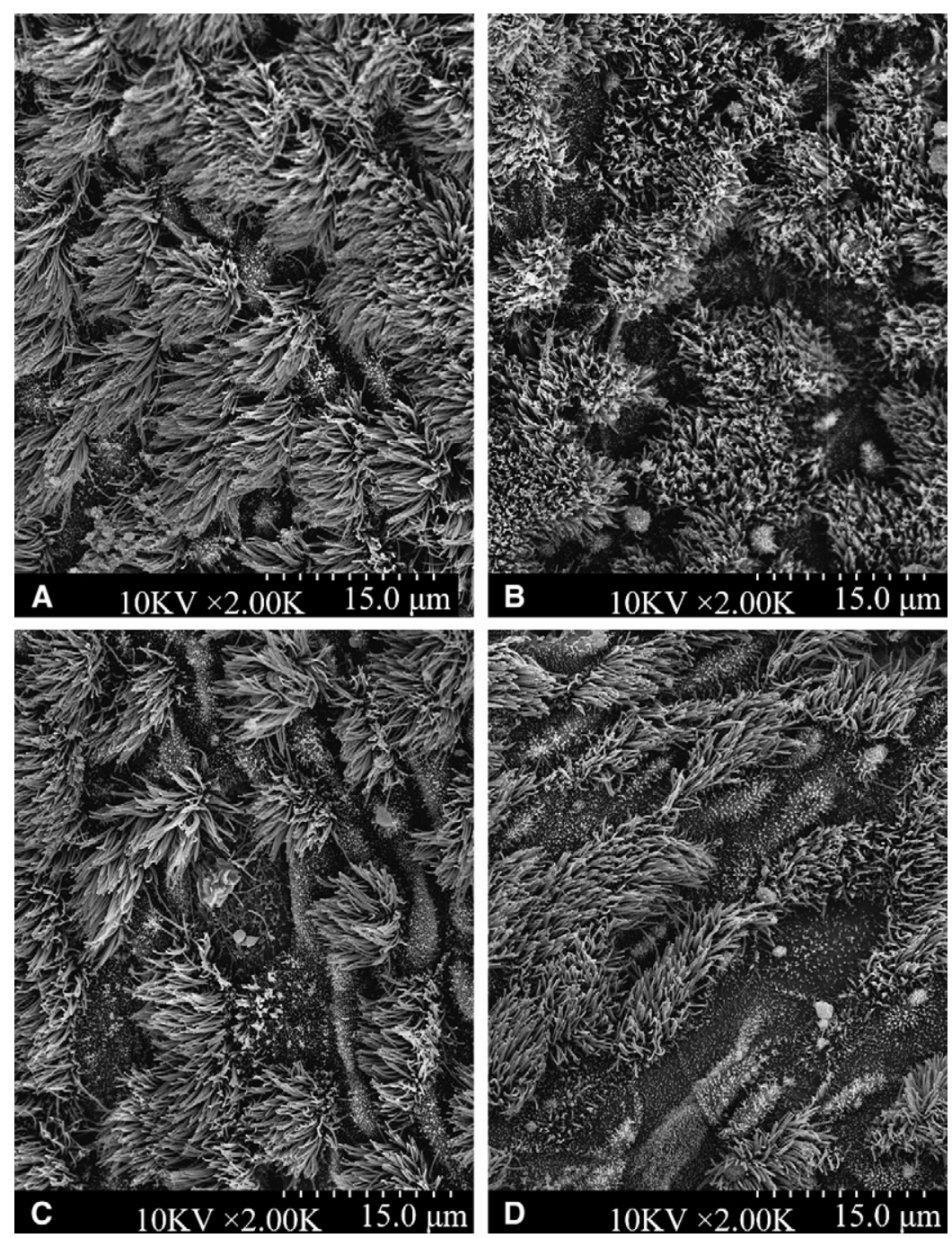

FIGURE 4. Fifty-two days after transplant, scanning electron microscopy showed epithelium in epidermal growth factor-loaded gelatin microsphere group (A) to be intact, well differentiated, and functional, whereas epithelial cells were relatively sparse in other groups. B, Epidermal growth factor group; C, gelatin microsphere group; D, control group.

EGF could promote airway epithelial cell proliferation, migration, and differentiation through both paracrine and autocrine paths. In 1993, Barrow and associates ${ }^{17}$ applied aerosolized EGF plus platelet-derived growth factor to sheep with acute airway injury, and these growth factors were shown to be effective in stimulating cell proliferation and accelerating the repair process. Kim and coworkers ${ }^{18}$ further demonstrated in vivo that the effects of EGF on repair of airway epithelium were concentration dependent and did not depend on the underlying matrix. Activation of EGF receptor by activators such as lower concentrations of lipopolysaccharide could accelerate wound repair of airway epithelium. ${ }^{19}$ Aria and assoicates ${ }^{20}$ found that the EGF messenger RNA expression was lower in the allografts than in the isografts in mouse heterotopic tracheal models. All these studies suggested that EGF might have a role in the reepithelialization of tracheal grafts, which is still under investigation.
Like other growth factors, EGF has a short half-life and requires a continuous exposure to the wounds to facilitate the regeneration, migration, and proliferation of epithelial cells. How to apply growth factors during tracheal transplant is a key problem. Ideally, to resolve this problem, first, growth factors should be applied persistently without interrupting the vitality of grafts; second, an effective concentration of growth factors should be maintained around the tracheal grafts; and third, the method should be simple and convenient. There have been many methods for administration of growth factors, such as direct local application, ${ }^{11}$ nebulization therapy, ${ }^{17}$ use of biomaterials as vectors, ${ }^{10,12-14}$ and gene therapy. ${ }^{15}$ These methods have their own disadvantages, however, and ideal methods of administration still remain to be achieved.

For topical application of EGF and maintenance of effective drug concentration in a local area for a long period, we 


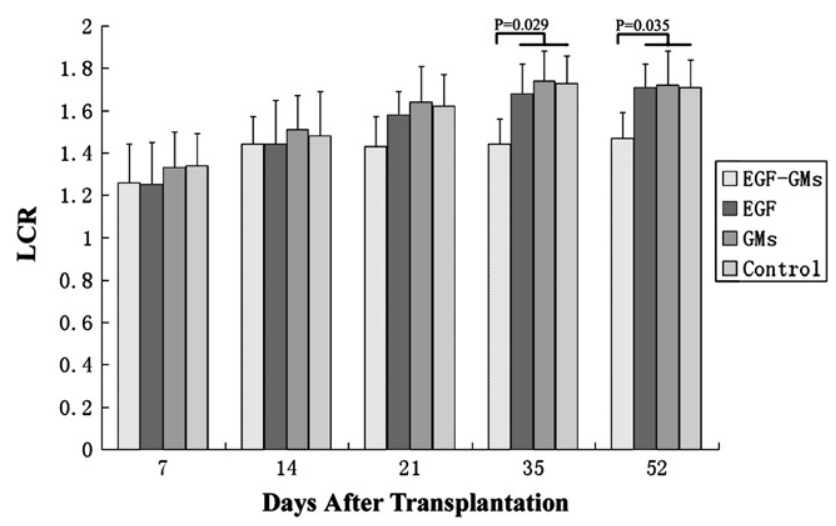

FIGURE 5. Subepithelial fibrosis was evaluated by ratio of lamina propria to tracheal cartilage $(L C R)$. On days 7, 14, and 21 after transplant, there was no statistically significant difference among groups. Ratio of group treated with epidermal growth factor-loaded gelatin microspheres ( $E G F-G M s$ ) was lower than ratios of groups treated with epidermal growth factor $(E G F)$, gelatin microspheres (GMs), or nothing (Control) on days 35 and 52. Error bars represent SD.

chose GMs as a vector to load EGF. Gelatin is nontoxic and biocompatible. It has been used clinically for hemostasis. Some researchers have been preparing gelatin as a vector to load biologic agents. ${ }^{14,21,22}$ Gelatin sheets or gelatin bilayer wound dressings containing EGF have been prepared to provide sustained-released EGF to accelerate wound repair. ${ }^{21}$ GMs could be made to load biologic agents according to the expansion characteristic and to release the agents with microsphere swelling and biodegradation. ${ }^{22}$ In this study, we prepared GMs as vectors to load EGF and provide sustained release. When applied locally to the grafts, these EGF-GMs could be concentrated around the tracheal graft. When the EGF-GMs were swollen and degraded, the EGF was persistently released. Our results showed that a dense population of ciliated epithelium appeared, and the ES was higher in the EGF-GM group on days 35 and 52, which suggests that the sustained release of EGF accelerated the reepithelialization of the tracheal grafts. The application of immunosuppressive agents could facilitate the regeneration of epithelium, ${ }^{6,23}$ which could have distorted our assessment of the effect of EGF on reepithelialization. To avoid the influence of immunosuppression, no immunosuppressive agents were used during this experiment.

EGF-GMs could facilitate airway epithelial regeneration and differentiation; therefore, they might accelerate fibrosis at the same time. In our study, we used LCR to evaluate the degree of fibrosis, because it has been used to evaluate the degree of fibrosis in many studies. ${ }^{6,24}$ Surprisingly, we found that fibrosis was not increased by EGF-GMs during the earlier phase after transplant (days 7, 14, and 21 after transplant). At the same time, the LCR in EGF-GM group was lower than that of other groups during later phase after transplant (days 35 and 52 after transplant). It is possible that the acceleration of reepithelialization may affect or even prevent the fibrosis to some degree. In our study, the LCR increased to a peak and maintained that level for a long time. The LCR in the EGF-GM group had reached a peak by day 14 . This peak was achieved earlier than that in the GM, EGF, and control groups. At the same time, the ES of the EGF-GM group began to increase rapidly. Murakawa and colleagues ${ }^{25}$ reported that efficient relining with recipient epithelium could prevent luminal fibrosis in murine tracheal grafts after retransplant into recipient strain mice. Thus the retardation of the fibrosis in this study may have resulted from the acceleration of reepithelialization. We also noticed that the LCR pattern in the EGF-GM group was different from that reported by Genden and associates. ${ }^{6}$ Their study showed that the LCR of nonimmunosuppressed orthotopic allografts increased and reached a peak by day 28 , whereas the LCRs of orthotopic isografts or immunosuppressed orthotopic allografts were low and remained consistent after transplant. In our study, the LCR of nonimmunosuppressed orthotopic allografts also increased, which was concordant with Genden and associates' study, ${ }^{5}$ but on days 35 and 52 the LCR of the EGF-GM group was lower than those of the other groups. Considering the difference between these 2 studies and the fact that reepithelialization of orthotopic tracheal grafts with recipient-derived epithelium may prevent rejection, ${ }^{4,5}$ we further speculate that the acceleration of reepithelialization may reduce fibrosis by reducing rejection.

In conclusion, we successfully prepared GMs loaded with EGF and applied them locally during tracheal transplant. Local administration of EGF increased the reepithelialization of the tracheal grafts. The acceleration of reepithelialization may reduce fibrosis through reduced immunologic rejection. Local application of EGF may be an effective method of accelerating epithelial regeneration of tracheal grafts in future clinical use. GMs.

We thank Dr Hong Wu for her contribution for preparing the

\section{References}

1. Jacobs JP, Quintessenza JA, Andrews T, Burke RP, Spektor Z, Delius RE, et al. Tracheal allograft reconstruction: the total North American and worldwide pediatric experiences. Ann Thorac Surg. 1999;68:1043-51.

2. Fernandez FG, Jaramillo A, Chen C, Liu DZ, Tung T, Patterson GA, et al. Airway epithelium is the primary target of allograft rejection in murine obliterative airway disease. Am J Transplant. 2004;4:319-25.

3. Genden EM, Iskander AJ, Bromberg JS, Mayer L. Orthotopic tracheal allografts undergo reepithelialization with recipient-derived epithelium. Arch Otolaryngol Head Neck Surg. 2003;129:118-23.

4. Genden EM, Govindaraj S, Chaboki H, Cleven H, Fedorova E, Bromberg JS, et al. Reepithelialization of orthotopic tracheal allografts prevents rejection after withdrawal of immunosuppression. Ann Otol Rhinol Laryngol. 2005;114:279-88.

5. Cleven HA, Genden EM, Moran TM. Reepithelialized orthotopic tracheal allografts expand memory cytotoxic T lymphocytes but show no evidence of chronic rejection. Transplantation. 2005;79:861-8.

6. Genden EM, Iskander A, Bromberg JS, Mayer L. The kinetics and pattern of tracheal allograft re-epithelialization. Am J Res Cell Mol Biol. 2003;28:673-81.

7. Hardwicke J, Schmaljohann D, Boyce D, Thomas D. Epidermal growth factor therapy and wound healing-past, present and future perspectives. Surgeon. 2008;6:172-7. 
8. Brown GL, Nanney LB, Griffen J, Cramer AB, Yancey JM, Curtsinger LJ 3rd, et al. Enhancement of wound healing by topical treatment with epidermal growth factor. $N$ Engl J Med. 1989;321:76-9.

9. Genden EM, Boros P, Liu J, Bromberg JS, Mayer L. Orthotopic tracheal transplantation in the murine model. Transplantation. 2002;73:1420-5.

10. Li X, Wang J, Ni Y, Yan X, Lu Q, Xu H, et al. Bone morphogenetic protein-2 stimulation of cartilage regeneration in canine tracheal graft. J Heart Lung Transplant. 2009;28:285-9.

11. Sung SW, Won T. Effects of basic fibroblast growth factor on early revascularization and epithelial regeneration in rabbit tracheal orthotopic transplantation. Eur J Cardiothorac Surg. 2001;19:14-8.

12. Nakanishi R, Nagaya N, Yoshimatsu T, Hanagiri T, Yasumoto K. Optimal dose of basic fibroblast growth factor for long-segment orthotopic tracheal autografts. J Thorac Cardiovasc Surg. 1997;113:26-36.

13. Govindaraj S, Gordon R, Genden EM. Effect of fibrin matrix and vascular endothelial growth factor on reepithelialization of orthotopic murine tracheal transplants. Ann Otol Rhinol Laryngol. 2004;113:797-804.

14. Igai H, Yamamoto Y, Chang SS, Yamamoto M, Tabata Y, Yokomise H. Tracheal cartilage regeneration by slow release of basic fibroblast growth factor from a gelatin sponge. J Thorac Cardiovasc Surg. 2007;134:170-5.

15. Krebs R, Tikkanen JM, Nykänen AI, Wood J, Jeltsch M, Ylä-Herttuala S, et al. Dual role of vascular endothelial growth factor in experimental obliterative bronchiolitis. Am J Respir Crit Care Med. 2005;171:1421-9.

16. Tikkanen JM, Hollmén M, Nykänen AI, Wood J, Koskinen PK, Lemström KB. Role of platelet-derived growth factor and vascular endothelial growth factor in obliterative airway disease. Am J Respir Crit Care Med. 2006; $174: 1145-52$.
17. Barrow RE, Wang CA, Evans MU, Herndon DN. Growth factors accelerate epithelial repair in sheep trachea. Lung. 1993;171:335-44.

18. Kim JS, McKinnis VS, Nawrocki A, White SR. Stimulation of migration and wound repair of guinea-pig airway epithelial cells in response to epithermal growth factor. Am J Respir Cell Mol Biol. 1998;18:66-74.

19. Koff JL, Shao MX, Kim S, Ueki IF, Nadel JA. Pseudomonas lipopolysaccharide accelerates wound repair via activation of a novel epithelial cell signaling cascade. J Immunol. 2006;177:8693-700.

20. Aria RM, Walsh S, Chalermskulrat W, Hathwar V, Neuringer IP. Growth factor upregulation during obliterative bronchiolitis in the mouse model. Am J Respir Crit Care Med. 2002;166:417-22.

21. Tanaka A, Nagate T, Matsuda H. Acceleration of wound healing by gelatin film dressings with epidermal growth factor. J Vet Med Sci. 2005;67:909-13.

22. Chen FM, Zhao YM, Wu H, Deng ZH, Wang QT, Zhou W, et al. Enhancement of periodontal tissue regeneration by locally controlled delivery of insulin-like growth factor-I from dextran-co-gelatin microspheres. J Control Release. 2006; 114:209-22.

23. Schrepfer S, Deuse T, Sydow K, Schäfer H, Detter C, Reichenspurner H. Tracheal allograft transplantation in rats: the role of different immunosuppressants on preservation of respiratory epithelium. Transplant Proc. 2006;38:741-4.

24. Kuo E, Bharat A, Shih J, Street T, Norris J, Liu W, et al. Role of airway epithelial injury in murine orthotopic tracheal allograft rejection. Ann Thorac Surg. 2006; 82:1226-33.

25. Murakawa T, Kerklo MM, Zamora MR, Wei Y, Gill RG, Henson PM, et al. Simultaneous LFA-1 and CD40 ligand antagonism prevents airway remodeling in orthotopic airway transplantation: implications for the role of respiratory epithelium as a modulator of fibrosis. J Immunol. 2005;174:3869-79. 